\title{
Measurements of three-dimensional flows induced by magnetic islands
}

\author{
C. M. Samuell $\odot,{ }^{1,}{ }^{*}$ J. D. Lore $\odot,{ }^{2}$ W. H. Meyer $\odot,{ }^{1}$ M. W. Shafer $\odot,{ }^{2}$ S. L. Allen, ${ }^{1}$ T. E. Evans $\odot,{ }^{3}$ and J. Howard ${ }^{4}$ \\ ${ }^{1}$ Lawrence Livermore National Laboratory, Livermore, California 94550, USA \\ ${ }^{2}$ Oak Ridge National Laboratory, Oak Ridge, Tennessee 37830, USA \\ ${ }^{3}$ General Atomics, San Diego, California 92186, USA \\ ${ }^{4}$ Australian National University, Canberra, ACT 0200, Australia
}

(Received 28 November 2018; revised manuscript received 21 May 2019; accepted 30 April 2020; published 4 June 2020)

\begin{abstract}
Magnetic reconnection and the resulting complex magnetic field geometries are fundamental aspects of our understanding of both space and laboratory plasmas. We report on the observation of three-dimensional (3D) impurity ion flow perturbation due to counterstreaming flows in the boundary plasma of the DIII-D tokamak. These counterstreaming flows arise not due to the magnetic reconnection itself but from the subsequent creation of collections of isolated flux tubes called magnetic islands. The magnetic islands form chains that wrap helically around the tokamak and modify local pressure balance generating a distinctive pattern of velocity perturbations that confirm long-standing 3D transport simulations. EMC3-EIRENE fluid modeling shows that these velocity perturbations arise from parallel pressure gradients that result principally from the radial temperature gradient across the magnetic islands and are supported by a momentum-loss feedback loop.
\end{abstract}

DOI: 10.1103/PhysRevResearch.2.023285

\section{INTRODUCTION}

The flow of charged particles streaming along magnetic field lines is at the heart of particle and energy transport in plasmas ranging from the solar corona to laboratory fusion devices. The perturbation of these field lines through magnetic reconnection can produce particle acceleration in its own right [1]; however, the altered shape of the magnetic field following a tearing and reconnection event also drives strong changes to ion dynamics. During reconnection, ions may couple to the reconnected field lines and get ejected into counterstreaming ion jets, a feature that has been used to identify reconnection in the solar wind and the Earth's magnetopause [2,3]. In this paper, a mechanism is described whereby counterstreaming ion flows are observed in a laboratory plasma due to the formation of magnetic islands in a tokamak device. While magnetic reconnection must occur for the islands to form, it is modification of the local pressure gradients that drives ion acceleration, not the reconnection itself.

Three-dimensional counterstreaming ion flows have been widely predicted in both stellarator and tokamak magnetic fusion devices where their presence affects neutral particle fueling, momentum transport, main-chamber erosion, and pressure balance [4,5]. In particular, this can occur around magnetic islands, regions of isolated magnetic flux [6] that

\footnotetext{
*samuell1@1lnl.gov

Published by the American Physical Society under the terms of the Creative Commons Attribution 4.0 International license. Further distribution of this work must maintain attribution to the author(s) and the published article's title, journal citation, and DOI.
}

form following reconnection, where distinct "streams" of particles flow inside and around the island in opposite directions. Figure 1 gives a conceptual idea of how islands can be positioned in a tokamak and how their presence modifies ion flows. While the existence of magnetic islands can generate a range of plasma instabilities in fusion devices, they can also be used as an engineering tool in stellarators to guide power and particles towards target plates that are equipped to handle high heat loads and to exhaust impurities, an idea that has been extensively modeled using the three-dimensional (3D) fluid modeling code EMC3-EIRENE [5]. The counterstreaming flows naturally arise around magnetic islands whereby particles flowing in opposite directions (toroidally) can interact. If sufficiently close to one another there will be a friction between the two streams that results in a loss of momentum in the direction perpendicular to the magnetic field. In the W7-AS and LHD stellarators, EMC3-EIRENE modeling revealed that this momentum loss can cause the experimentally observed reduction in ion target current before onset of a high-recycling regime due to the associated modification of the pressure balance [5,7]. Recently their presence on the W7-X stellarator has been confirmed and compared to EMC3-EIRENE modeling which highlighted the role of drift effects in determining scrape-off layer behavior [8]. While additional momentum loss and a reduced threshold for detachment in island diverted stellarators is beneficial from the perspective of power exhaust, the lack of a high-recycling regime increases the challenge of finding acceptable particle control solutions.

Characterization of these flows is challenging due to their inherent 3D nature and often small spatial scales; however, there have been observations of counterstreaming flows using Mach probes and traditional line-integrated spectroscopy in stellarators $[9,10]$ in addition to the recent coherence 
(a)
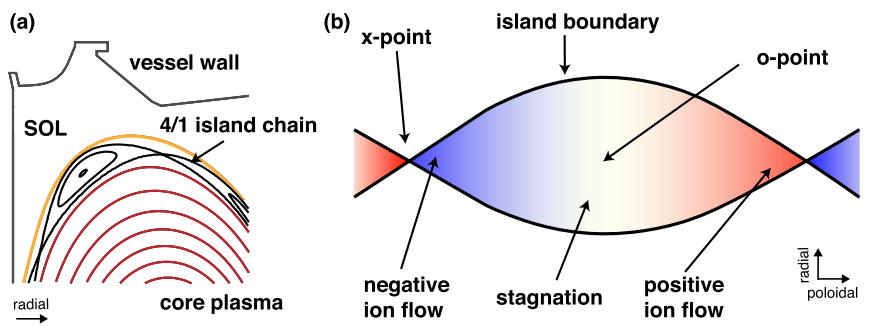

FIG. 1. Schematic showing how magnetic islands can perturb (a) the tokamak magnetic field configuration and (b) the direction ions flow along field lines inside the island.

imaging results in $\mathrm{W} 7-\mathrm{X}$ [8]. There is also a range of works examining the change in tokamak core-plasma rotation due to magnetic islands [11-13]. In this paper, we image fully $3 \mathrm{D}$ ion flows in the boundary plasma of a tokamak using resonant magnetic fields to generate the 3D magnetic topology.

\section{EXPERIMENTAL OBSERVATION}

To investigate the interaction between magnetic islands and ion flows, experiments were carried out on the DIII-D tokamak . A deuterium inner-wall-limited and low-confinement discharge with large coherent island chains was produced. These island chains were generated by applying a nonaxisymmetric resonant magnetic field perturbation (RMP) to an otherwise nominally axisymmetric plasma [14]. The resulting island chains had a toroidal mode number of $1(n=1)$ indicating a single island chain extends one period toroidally, but multiple periods poloidally ( $m=2$ in the core, extending out to $m=4$ near the edge of the core plasma). These mode numbers are set by the phase and amplitude in the 3D coil set, and verified using magnetic probes. Doppler coherence imaging spectroscopy (CIS) was used to measure $\mathrm{C}^{2}+$ impurity velocities throughout the boundary plasma, referred to as the scrape-off layer (SOL). This diagnostic technique is an alternative to traditional velocity measurements that can be used for imaging the spectral qualities of plasma species emitting in the visible spectrum [15]. The imaging nature of CIS gives it an advantage over other ion velocity measurement techniques for investigating complex phenomena as the extension from 2D to 3D measurement is straightforward; stationary magnetic structures can be rotated through the camera's field of view as a function of time for a 3D characterization. Two CIS systems are installed on DIII-D [16,17] that measure the bright $C^{2}+$ ion emission $(465 \mathrm{~nm})$ with a viewing geometry that results in a measurement of mostly toroidally directed velocities. Measurements can be made wherever there is sufficient density of $\mathrm{C}^{2}+$ ions and the electron temperature is high enough for electron excitation of the ion, but low enough that ionization to higher charge states does not occur too readily, although this can be influenced by transport effects. In practice this results in the majority of light coming from the vicinity of $T_{e} \sim 8 \mathrm{eV}$.

The time history of a DIII-D shot displaying the emergence of magnetic islands is presented in Fig. 2 where five individual time periods are identified [(i)-(v)]. This was an $\sim 4 \mathrm{MW}$ discharge with toroidal magnetic field $B_{T}=1.95 \mathrm{~T}$ (ion $B \times \nabla B$ down), plasma current $I_{p}=1.28 \mathrm{MA}$, line-

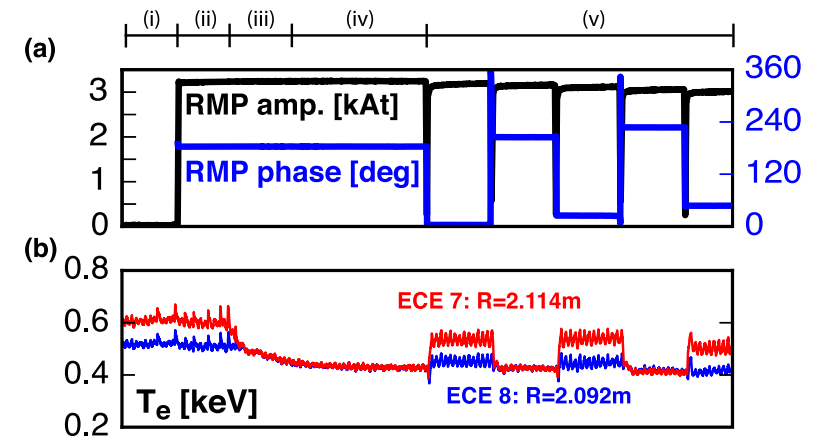

(c)

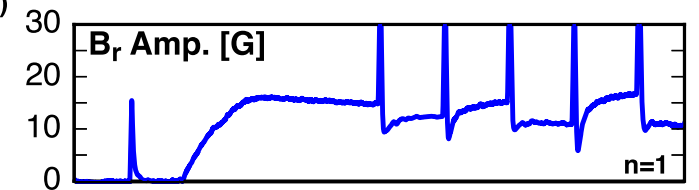

(d)

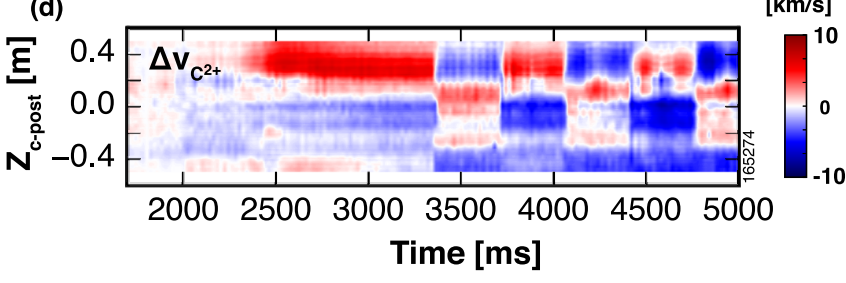

FIG. 2. Discharge evolution as $n=1$ islands form, and are moved toroidally, including (a) amplitude and phase of applied RMP; (b) electron temperature at two ECE chord radial positions $(<2 \%$ relative error between channels, $5 \%$ absolute); (c) $n=1$ component of radial magnetic field plasma response ( $\sim 2 \%$ error); and (d) change in line of sight $\mathrm{C}^{2}+$ velocity along the center post with respect to the velocity at $1800 \mathrm{~ms}$ (pre-RMP) ( $<1 \mathrm{~km} / \mathrm{s}$ absolute error). Discrete time periods in shot correspond to (i) axisymmetric plasma (2D), (ii) RMP energized, (iii) RMP field penetration causing island creation and locking, (iv) RMP saturation, and (v) phase flips to move islands with respect to stationary diagnostics.

averaged density of $\overline{n_{e}} \sim 4 \times 10^{19} \mathrm{~m}^{-3}$, and a RMP produced using 3D coils external to the machine (C coils, $3.4 \mathrm{kAt}$ peak [18]). The presence of magnetic islands in the core is confirmed by combining observation of the flattening of electron temperature across the $2 / 1$ island chain using two radially separated electron cyclotron emission (ECE) chords [Fig. 2(b), [19]] and the rise in $n=1$ radial magnetic field response that occurs as the RMP penetrates [Fig. 2(c), [20]]. The SOL flow velocity response to the islands is shown in Fig. 2(d) where the vertical variation of the tangential velocity averaged across a 2 -cm-wide radial region of interest (ROI) along the center post. A key aspect of this work is that coherent islands do not appear in the SOL; instead, CIS is measuring the impact of "core" islands perturbing the SOL. The change in velocity, $\Delta v$, in the ROI is referenced to a 2D time slice $(1800 \mathrm{~ms})$ to emphasize temporal changes and remove systematic experimental uncertainties. As the islands emerge, positive and negative velocity perturbations develop along the center post. Changing the phase of the RMP rotates the islands through the camera's field of view (poloidally) and gives rise to an associated change in both the sign and magnitude of the velocity perturbation that demonstrates the 3D nature of the perturbation. 


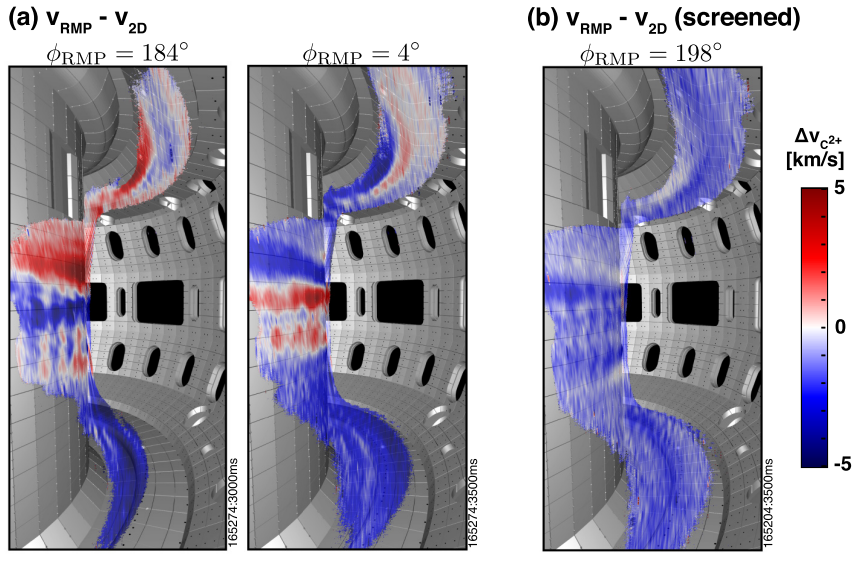

FIG. 3. (a) Images of line-integrated (mostly toroidal) $\mathrm{C}^{2}+$ velocities referenced to the axisymmetric part of the discharge (preRMP) at particular applied RMP phases showing a pattern of positive and negative velocity perturbation. Subplot (b) is for a case where islands did not form despite the presence of 3D fields. A partial transparency has been added to aid comparison with the CAD model.

The full effect of the islands on the SOL flow pattern can be seen by examining the 2D CIS velocity images as shown in Fig. 3. Panel (a) shows the change in flow velocity due to the magnetic islands at two phases $180^{\circ}$ apart corresponding to times 3 and $3.5 \mathrm{~s}$ in Fig. 2. Line-of-sight-integrated velocities are shown in regions where $\mathrm{C}^{2}+$ brightness was sufficiently high that the measurement could be made and masked elsewhere. Note that while islands are formed in the core, these CIS measurements are in the SOL and so our focus is on the impact of the presence of the islands rather than imaging islands directly. Here it can be seen that a poloidally alternating pattern of positive and negative flow perturbation extends from the limiting surface to the far SOL in the top of the machine. Reversing the sign of the RMP phase causes a reversal in the perturbation's sign across most of the measurement region. This sign change corresponds to imaging the opposite part of the $n=1$ island chain (x points exchanged for o points) with accompanying change in toroidal direction of the flows. This effect is more pronounced in the top of the machine which displayed a stronger response to the 3D perturbation than was observed in the bottom of the machine, possibly due to the inherent asymmetry of cross-field drifts. Changes in $\mathrm{C}^{2}+$ line-of-sight-integrated velocity were as high as $\pm 6 \mathrm{~km} / \mathrm{s}$ compared to an unperturbed flow velocity maximum of about $20-30 \mathrm{~km} / \mathrm{s}$.

That the flow perturbations are associated with island formation (as opposed to simply the 3D fields) is confirmed by examining a case where rotational screening limited the penetration of the imposed 3D fields so that islands do not form. Velocity measurement from this screened case showed that while some changes in SOL flows are observed, the magnitude is about five times lower than when islands were present and the characteristic poloidally alternating pattern is substantially less pronounced as seen in Fig. 3(b). In this case there is an absence of strong positive perturbation and an overall drift towards negative values that was observed in both screened and nonscreened cases. In the instance where 3D
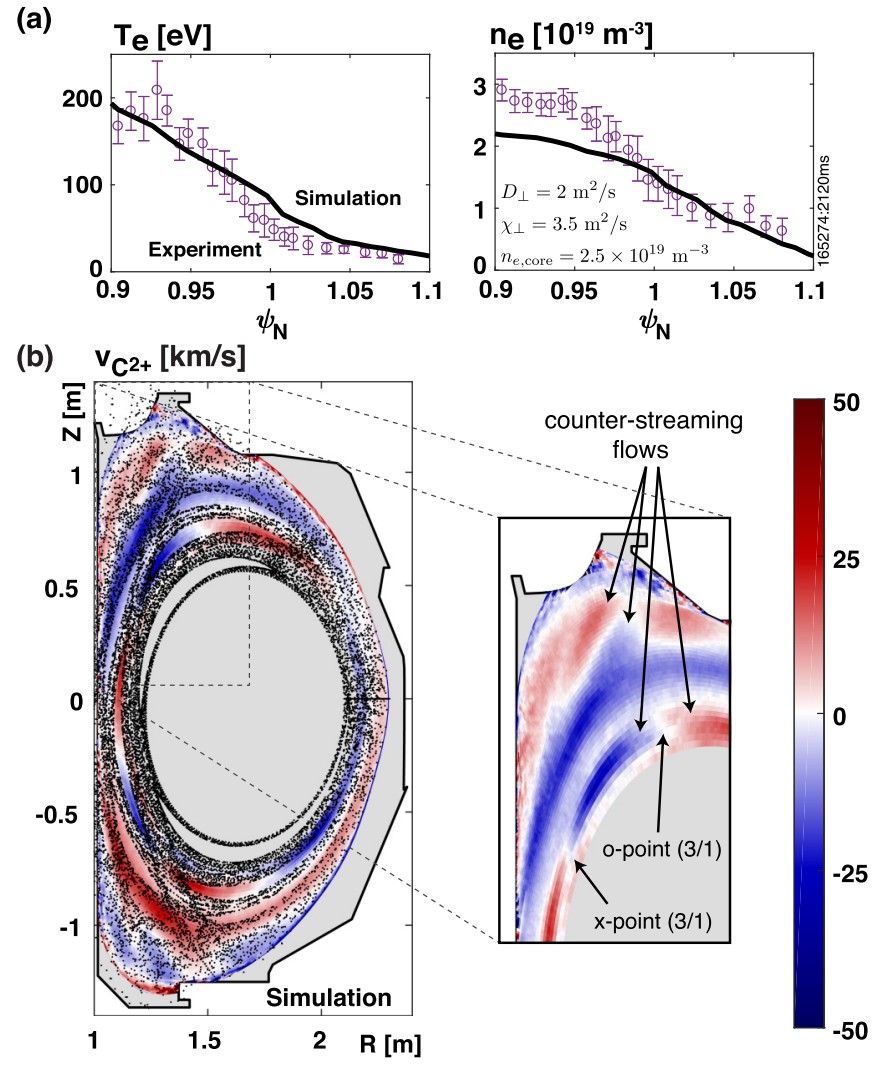

FIG. 4. (a) Density and temperature profiles measured experimentally (circles) and simulated (lines). (b) Simulated $C^{2}+$ parallel velocity at a toroidal slice of $\phi=-120^{\circ}$. Velocity contours are overlaid with a Poincaré plot (dots) to show magnetic island positions (absence of dots). Note $\mathrm{C}^{2}+$ velocity is plotted throughout the simulation domain including regions where emissivity may not be high enough to view with CIS.

fields are active but no islands form we would expect a more stochastic boundary than in the resonant case where islands form.

\section{COMPARISON WITH SIMULATIONS}

The 3D fluid-plasma edge transport code EMC3EIRENE [21,22] has been used to model these discharges to gain intuition on the physics behind the experimentally observed flow perturbations. While counterstreaming flows in the main-ion population have been widely predicted, ascertaining the behavior of impurity species is more complex given the need to consider plasma-wall interactions, neutral recycling, and the impurity-specific force balance. The EMC3 code is used to solve the fluid transport equations for particles, parallel momentum, and energy in a three-dimensional geometry and is coupled to EIRENE, which provides kinetic neutral particle recycling and transport. Particle and heat diffusivities that determine cross-field transport were chosen to match Thompson scattering profiles [Fig. 4(a) and inset] matched at a time slice after 3D coils had been energized but before the emergence of islands $(2120 \mathrm{~ms})$. A vacuum approximation was used to calculate the effect of the RMP that sums contributions from the coils and plasma current 

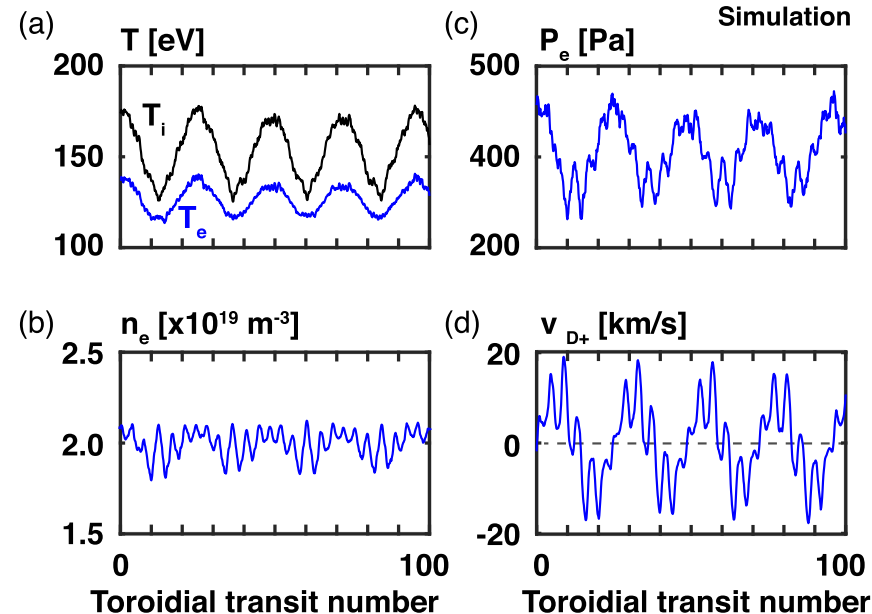

FIG. 5. Simulation quantities along 100 toroidal transits of a field line located near the separatrix of the $4 / 1$ island chain. Variations in electron (blue) and ion (black) temperature (a) combine with density (b) to generate a changing parallel pressure (c) along magnetic field lines which manifests as a changing parallel ion velocity (d).

but does not include the plasma response [23]. $\mathrm{C}^{2}+$ impurity parallel velocity profiles from these simulations are shown in Fig. 4(b) where it can be seen that counterstreaming impurity flows are predicted inside islands, particularly the $3 / 1$ island chain in the core plasma. In the SOL, effects originating from the counterstreaming flows and $3 \mathrm{D}$ perturbations will be convolved with the complex set of forces that balance to drive SOL behavior in 2D plasmas.

Our simulations show parallel pressure gradients are the physical mechanism that drives these 3D flows. To illustrate this, the conditions along a field line at the outside surface of the 4/1 island chain for 100 toroidal transits is shown in Fig. 5; a core example is examined first for simplicity. During these transits the field line is sampling both the insideand outside-facing surfaces of the island (radially) as well as the small and large major radius sides of the machine as it maps out the entire island chain. Changes in temperature along the field line result from sampling either the radial inside or outside of the island (i.e., the small or large minor radius island surface). It is noted that while the temperature profile flattens due to the presence of the magnetic island, it is not entirely flat radially; the inside of the island remains hotter than the outside. Density variations stem from the field line's traversal from the localized ionization region near the center post to the large major radius side of the machine where the density is lower. These density and temperature gradients combine to generate a varying pressure along the field line. The pressure is periodic with the low-frequency component coming from the temperature variation while the high-frequency component is from the density variation. This pressure gradient produces a force that acts on the ion velocity and so is responsible for the changing $\mathrm{D}^{+}$parallel velocity shown in Fig. 5(d) which rises and falls in phase with the pressure, i.e., the strong pressure gradients result in ion acceleration and accompanying fast parallel velocities. Where there is no gradient in pressure the ion flow stagnates; for example, at the island o points. The situation is more complex in the SOL where 3D features including connection length modification and effects due to the islands are present but are also folded in with the primarily 2D phenomena driving SOL flows and impacted by transport across the core-edge boundary. For example, pressure gradients along open field lines drive particles to flow between regions of high local ionization (particle source) and the walls (particle sink). The islands will dominate flow behavior in the core and the requirement that ions be at the sound speed will dominate at the edge; in the SOL between these two cases the connection length and cross-field momentum transfer will be important. The net effect in the SOL is a perturbation of the background axisymmetric flows, that could lead, for example, to a localized reduction in the parallel flow velocity of impurities towards the divertor target plates. When numerous regions of counterstreaming flows are present, there is a deviation from total pressure conservation along field lines where $p_{\text {tot }}=m n v^{2}+n_{e} T_{e}+n_{i} T_{i}$. This deviation is caused by a pressure loss due to cross-field momentum transport in the model which captures the momentum loss that occurs from having counterstreaming flows in close spatial proximity. For the $3 \mathrm{D}$ flows generated by islands to propagate outside the confined islands into the SOL requires a significant pressure loss from perpendicular viscosity.

Care must be taken in using a numerical model to understand experimental measurements. However, this modeling work presents a physical mechanism by which our experimental observation of 3D perturbation in impurity flows in the far SOL would imply the presence of momentum loss due to counterstreaming flows. In an axisymmetric plasma, significant electron or ion parallel pressure gradients such as those shown in Fig. 5(d) would be rapidly dissipated by parallel flows. However, in the 3D case described here, the pressure gradients cannot relax because of the dissipation of the momentum term in $p_{\text {tot }}$ resulting from the counterstreaming flows; a feedback loop is established whereby the ion and electron pressure gradients drive counterstreaming flows and the momentum loss from these same counterstreaming flows allows the ion and electron pressure gradients to be maintained.

To make a direct comparison between the experimental and simulated 3D flows, a synthetic diagnostic was developed to predict the velocities CIS would expect to see given the EMC3-EIRENE simulation solution. This synthetic diagnostic calculates the line integrals of emissivity-weighted velocity throughout the EMC3-EIRENE grid (Fig. 6). The principal uncertainty in this technique is the interaction between the optical and tokamak geometry which was minimized by incorporating physical measurement of optical components into the tokamak CAD description that were subsequently optimized by minimizing the error on the tomographic reconstruction technique more routinely applied in 2D axisymmetric discharges. The experimentally observed pattern of velocity perturbation also appears in the simulations indicating the fundamental 3D structure, and features are similar in both cases. Some individual striations appear at different poloidal locations, which may be due to the charge-state localization of $\mathrm{C}^{2}+$ emission. The maximum difference between the two phases of about 4 and $10 \mathrm{~km} / \mathrm{s}$ in the simulation and experiment, respectively, indicate a quantitative difference not dissimilar to the two to three times discrepancy that has been 
(a) Simulation

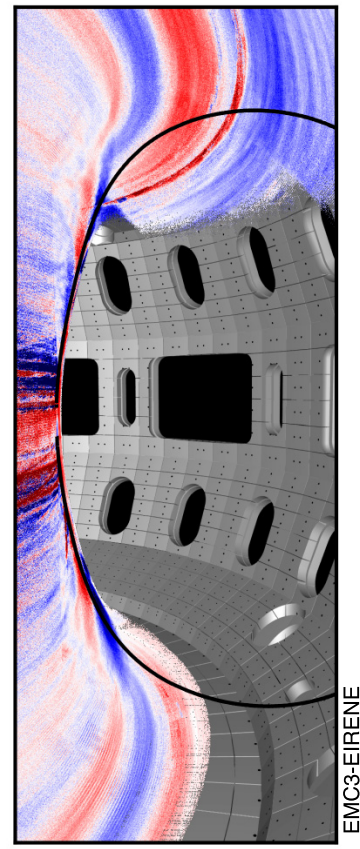

\section{(b) Experiment}

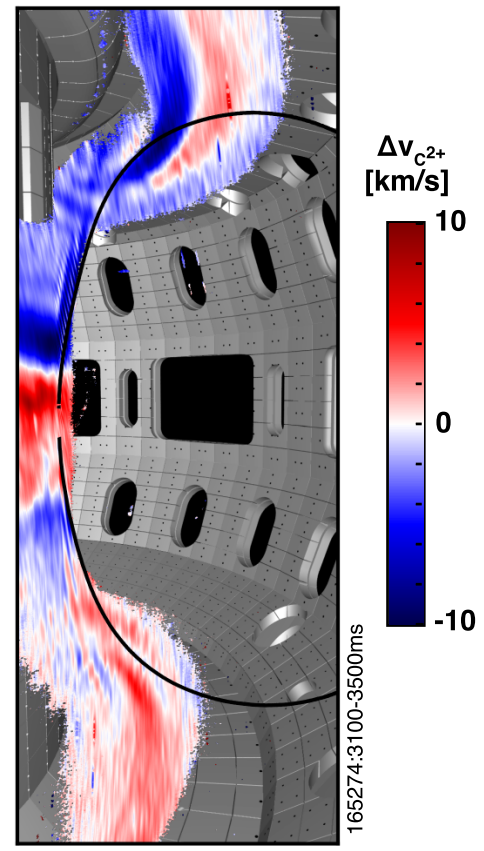

FIG. 6. Comparison of simulated (a) and experimentally measured (b) line-integrated $\mathrm{C}^{2}+$ velocities where the velocity displayed is the difference between two toroidal phases separated by $180^{\circ}$. Note the presence of a striation pattern in both cases. The axisymmetric last closed flux surface projected onto the camera's tangency plane is also shown for reference (black line).

observed in 2D comparisons [16,24,25]. This quantitative comparison may be improved with the inclusion of additional physics such as kinetic effects, turbulent particle transport, cross-field drifts, and the plasma response to the applied RMP.

\section{SUMMARY}

3D SOL flows around magnetic islands predicted by EMC3-EIRENE modeling have been imaged in a tokamak and directly compared to simulations. Simulations show that perpendicular temperature gradients generate parallel pressure gradients due to the inherently 3D nature of the magnetic island geometry. Ion transport along field lines responds to this pressure gradient, establishing regions of ion acceleration away from pressure peaks, ultimately forming counterstreaming flows. The cross-field momentum loss from counterstreaming flows causes pressure to no longer be conserved along field lines, and a feedback loop forms whereby the 3D flows contribute to maintaining the pressure gradients that are themselves responsible for the complex flow pattern observed. These effects must be considered both in interpreting data from 3D laboratory and space plasma reconnection events as well as for predicting behavior in the boundary of nextgeneration magnetic fusion devices.

\section{ACKNOWLEDGMENTS}

This material is based upon work supported by the US Department of Energy, Office of Science, Office of Fusion Energy Sciences, using the DIII-D National Fusion Facility, a DOE Office of Science user facility, under Awards No. DE-FC02-04ER54698, No. DE-AC52-07NA27344, and No. DE-AC05-00OR22725.

This report was prepared as an account of work sponsored by an agency of the United States Government. Neither the United States Government nor any agency thereof, nor any of their employees, makes any warranty, express or implied, or assumes any legal liability or responsibility for the accuracy, completeness, or usefulness of any information, apparatus, product, or process disclosed, or represents that its use would not infringe privately owned rights. Reference herein to any specific commercial product, process, or service by trade name, trademark, manufacturer, or otherwise, does not necessarily constitute or imply its endorsement, recommendation, or favoring by the United States Government or any agency thereof. The views and opinions of authors expressed herein do not necessarily state or reflect those of the United States Government or any agency thereof.
[1] G. Paschmann, B. U. Ö. Sonnerup, I. Papamastorakis, N. Sckopke, G. Haerendel, S. J. Bame, J. R. Asbridge, J. T. Gosling, C. T. Russell, and R. C. Elphic, Nature (London) 282, 243 (1979).

[2] T. D. Phan, L. M. Kistler, B. Klecker, G. Haerendel, G. Paschmann, B. U. Ö. Sonnerup, W. Baumjohann, M. B. Bavassano-Cattaneo, C. W. Carlson, A. M. DiLellis, K.-H. Fornacon et al., Nature (London) 404, 848 (2000).

[3] J. T. Gosling, R. M. Skoug, D. J. McComas, and C. W. Smith, J. Geophys. Res.: Space Phys. 110, A01107 (2005).

[4] H. Frerichs, O. Schmitz, T. Evans, Y. Feng, and D. Reiter, Phys. Plasmas 22, 072508 (2015).

[5] Y. Feng, F. Sardei, P. Grigull, K. McCormick, J. Kisslinger, and D. Reiter, Nucl. Fusion 46, 807 (2006).

[6] F. Waelbroeck, Nucl. Fusion 49, 104025 (2009).
[7] M. Kobayashi, Y. Xu, K. Ida, Y. Corre, Y. Feng, O. Schmitz, H. Frerichs, F. L. Tabares, T. E. Evans, J. W. Coenen, Y. Liang et al., Nucl. Fusion 55, 104021 (2015).

[8] V. Perseo, F. Effenberg, D. Gradic, R. König, O. P. Ford, F. Reimold, D. A. Ennis, O. Schmitz, T. S. Pedersen, and the W7-X Team, Nucl. Fusion 59, 124003 (2019).

[9] M. Kobayashi, S. Morita, and M. Goto, Rev. Sci. Instrum. 88, 033501 (2017).

[10] A. R. Akerson, A. Bader, C. C. Hegna, O. Schmitz, L. A. Stephey, D. T. Anderson, F. S. B. Anderson, and K. M. Likin, Plasma Phys. Controlled Fusion 58, 084002 (2016).

[11] T. Evans, Plasma Phys. Controlled Fusion 57, 123001 (2015).

[12] E. Taylor, C. Cates, M. Mauel, D. Maurer, D. Nadle, G. Navratil, and M. Shilov, Phys. Plasmas 9, 3938 (2002).

[13] L. Eliseev, N. Ivanov, A. Kakurin, A. Melnikov, and S. Perfilov, Phys. Plasmas 22, 052504 (2015). 
[14] T. E. Evans, R. A. Moyer, J. G. Watkins, P. R. Thomas, T. H. Osborne, J. A. Boedo, M. E. Fenstermacher, K. H. Finken, R. J. Groebner, M. Groth, J. Harris et al., J. Nucl. Mater. 337-339, 691 (2005).

[15] J. Howard, J. Phys. B: At., Mol. Opt. Phys. 43, 144010 (2010).

[16] C. M. Samuell, G. D. Porter, W. H. Meyer, T. D. Rognlien, S. L. Allen, A. Briesemeister, A. G. Mclean, L. Zeng, A. E. Jaervinen, and J. Howard, Phys. Plasmas 25, 056110 (2018).

[17] C. M. Samuell, S. L. Allen, W. H. Meyer, R. C. Isler, A. Briesemeister, R. S. Wilcox, C. J. Lasnier, A. G. Mclean, and J. Howard, Rev. Sci. Instrum. 89, 093502 (2018).

[18] T. Evans, R. Moyer, and P. Monat, Phys. Plasmas 9, 4957 (2002).

[19] M. Austin and J. Lohr, Rev. Sci. Instrum. 74, 1457 (2003).
[20] E. Strait, Rev. Sci. Instrum. 77, 023502 (2006).

[21] Y. Feng, F. Sardei, J. Kisslinger, and P. Grigull, J. Nucl. Mater. 241-243, 930 (1997).

[22] Y. Feng, F. Sardei, and J. Kisslinger, J. Nucl. Mater. 266-269, 812 (1999).

[23] J. D. Lore, A. R. Briesemeister, N. M. Ferraro, H. Frerichs, B. Lyons, A. McLean, J.-K. Park, and M. W. Shafer, Nucl. Fusion 57, 056025 (2017).

[24] S. Erents, R. Pitts, W. Fundamenski, J. Gunn, and G. Matthews, Plasma Phys. Controlled Fusion 46, 1757 (2004).

[25] M. Groth, J. A. Boedo, N. H. Brooks, R. C. Isler, A. W. Leonard, G. D. Porter, J. G. Watkins, W. P. West, B. D. Bray, M. E. Fenstermacher, R. J. Groebner et al., Nucl. Fusion 49, 115002 (2009). 